\title{
Inter-organization Cooperation for Care of the Elderly
}

\author{
Ricardo Costal, Paulo Novais2, José Machado2 , Carlos Alberto3 and José \\ Neves2 \\ 1 College of Management and Technology - Polytechnic of Porto, \\ Felgueiras, Portugal rfe@estgf.ipp.pt \\ 2 DI-CCTC, Universidade do Minho, Braga, Portugal \\ \{pjon,jmac,jneves\}@di.uminho.pt \\ 3 Hospital Geral de Santo António, EPE, Porto, Portugal \\ calberto.admn@hgsa.min-saude.pt
}

\begin{abstract}
With the growing numbers of the elderly population, the society is face to face with a set of new problems, namely the lack of resources to assist their living in a noble mode. Nevertheless, with the use of new computational technologies and novel methodologies for problem solving, some solutions to these problems are emerging (e.g., remote sensing/assistance/supervision). Therefore, it is our goal to show that under such scenarios, it is possible to bring into play different interconnected virtual organizations, through which will be provided to the population, in general, and the elderly, in particular, a number of services (e.g., healthcare, entertainment, learning), without delocalization or messing up with their routine.
\end{abstract}

\section{Introduction}

Once the human population is progressively ageing, it matters that the elderly in need of special attention, is growing. Old age brings new problems (e.g. entertainment, health, lowliness), aggravated with the lack of specialized human resources to assist their necessities. Besides that fact, one may point out, for example, that pressure exists in government and society (e.g. budgetary restraints, cost of medical technologies and cost of internment) that will force readjustments of actual entertainment and/or health care practice, which may also affect other co-related public systems [1,2].

This work looks at the role that inter-organization cooperation and learning plays within the innovative processes of a smart home for care of the elderly, and, suggests a framework that allows a set of organizations to strategically model a collaborative

Please use the following format when citing this chapter:

Costal, R., Novais, P., Machado, J., Alberto, C., Neves, J., 2007, in IFIP International Federation for Inlormation Processing, Volume 252, Integration and Innovation Orient to L-Society Volume 2, eds. Wang, W., (Boston: Springer), pp. 200-208 
environment that is conducive to innovation. The major idea is to enhance elderly quality of life, allowing them an "active ageing", thus being able to participate in social, economic, cultural, spiritual, civic and family affairs, physically and labor, remaining active contributors to their families and communities [1]. The path to pursue, in order to achieve the presented idea, relies on a mix of different sensibilities from Artificial Intelligence, such as Decision Trees and Automated Learning, coupled whit different computational paradigms and methodologies for problem solving, such as Agent Based Systems and Group Decision Support Systems [3], thus being able to achieve a high level of "intelligence" in what may be denominated as "Smart Healthcare Homes" [4].

\subsection{Inter-organization Cooperation}

In Inter-Organization Cooperation there are factors that tend to be surrounded in the local milieu, which according to Dosi [5], can be seen as the social embedded processes that allow organizations to obtain outside complementary knowledge and be innovative in the course of interaction among different actors, i.e., the local or regional milieu needs to include not only the substances related to the service structure or economics terms, but also social, cultural and institutional ones [6]. Thus, in the interaction of the different actors, the cooperation elements can be found in a kind of common language, social relationship, norms, values and institutions, which in our work will be set in terms of an extension to the logic programming language, being their knowledge bases built as logical theories that found their foundations on this extension [7]. Conclusions are supported by deductive proofs, or by arguments that include conjectures and motivate new topics of inquiry, i.e., if deduction is fruitless the agent inference engine resorts to abduction, filling in missing pieces of logical arguments with plausible conjectures to obtain answers that are only partly supported by the facts available (to the inference engine).

\section{Business Integration for Healthcare}

Our objective is to present an intelligent multi-agent system that will be able to monitor, interact and serve its costumers, being those elderly people and/or their relatives. This system will be interconnected, not only to healthcare institutions, but also with leisure centers, training facilities, shops and relatives, just to name a few. The VirtualECare Architecture is a distributed one with different components interconnected through a network (e.g. LAN, MAN, WAN), each one with a different role (Fig).

This solution helps healthcare providers to integrate, analyze, and manage complex and disparate clinical, research and administrative knowledge. It provides tools and methodologies for creating an information-on-demand environment that can improve quality-of-living, safety, and patient care. 


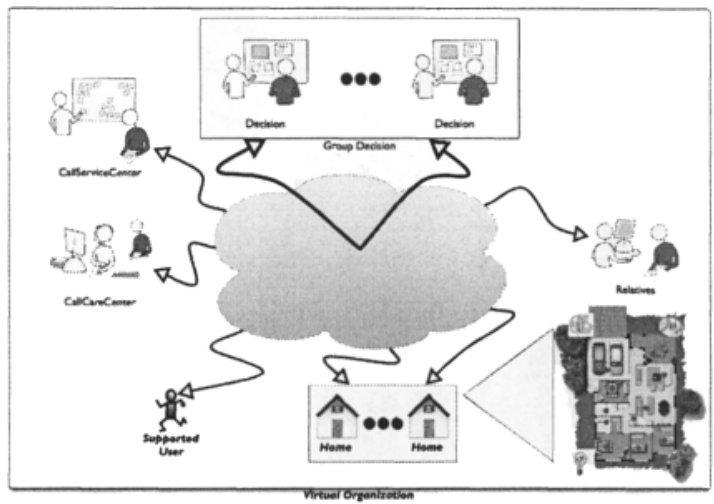

Fig 1. The System's Architecture, VirtualECare

\section{Decision Making}

To model the call centers it is necessary to establish the steps for the creation of a virtual community of agents, i.e. a Multi Agent System (MAS). The importance of maintaining a community of agents is directly related to the need of obtaining information, among others, about their credibility, reputation, as well as their past behaviors, i.e.:

Agent(Id)::area_of_expertise $\wedge$ organizational_factors $\wedge$ interest_topics $\wedge$ disponibility $\wedge$ credibility $\wedge$ reputation $\wedge$ availability.

where Id, area_of_expertise, organizational factors, interest_topics, disponibility, credibility, reputation and availability denote, respectively, the identification of the agent, the set of areas where the agent is an expert, the information about the institution where the agent is enrolled (e.g. employee numbers), the interest topics for the agent, the disponibility, credibility, reputation of the agent and its availability at a given moment. The community of agents is given as a set of $\mathrm{N}$ agents, $\left\{\mathrm{AgP}_{1}, \mathrm{AgP}_{2}, \ldots \mathrm{AgP}_{\mathrm{N}}\right\}$, denoted by $\mathrm{AgP}$. The availability of each agent can be classified according to three states: uncommitted, committed, or in action. An uncommitted agent stands for someone that may or may not join the MAS. A committed agent has agreed to be part of the MAS, but the inclusion process has not yet started. At last, an agent in action is someone involved in a task that already began. 


\subsection{Incomplete Information}

The agent's KB has two different types of knowledge: the positive knowledge (that is known to be true), and the negative one (that is known to be false). All the rest is unknown [7]. Indeed, the view of logic programming accepted in this paper is strictly declarative. The adequacy of a body representation of knowledge in a logic programming language means adequacy with respect to the declarative semantics of that language. Given a First Order Language (FOL), an Extended Logic Program (ELP) is a set of rules and invariants of the form:

$$
H \leftarrow B_{1} \wedge \ldots \wedge B_{n} \wedge \neg C_{1} \wedge \ldots \wedge \neg C_{m}(m>0, n>0)
$$

where $H, B_{1}, \ldots, B_{n}, C_{1}, \ldots, C_{m}$ are objective literals, and, in integrity rules, $H$ is $\perp$ (contradiction). An objective literal is either an atom $\mathrm{A}$ or its explicit negation $\neg A$, where $\neg \neg A=A . \neg L$ is called a default or negative literal. Literals are either objective or default ones. The default complement of objective literal $L$ is $\neg L$, and of default literal $\neg L$ is $L$. A rule stands for all its ground instances wrt (with respect to) FOL. A set of literals $S$ is non-contradictory iff there is no $L \in S$ such that $\neg L \in S$. For every pair of objective literals $\{\mathrm{L}, \neg \mathrm{L}\}$ in FOL we implicitly assume the integrity rule $\perp \leftarrow L, \neg L$. The main idea here is to compute all consequences of the program, even those leading to contradiction, as well as those arising from contradiction. Suppose that in the $\mathrm{KB}$ of the $\mathrm{AgR}$ the information related to the areas of expertise of the $\mathrm{AgP}$; identified as Peter, is represented in Program 1 [7].

area_of_expertise('Peter', pediatrics).

$\neg$ area_of_expertise('Peter', oncologist).

Program 1. It contains information related to the expertise areas of a specific agent.

If the KB is questioned if the area of expertise of Peter is Pharmacy the answer should be unknown, because there is no information related to that. On other hand, situations of incomplete information may involve different kinds of nulls. The ELP language will be used for the purpose of knowledge representation. One of the null types to be considered stands for an unknown value, a countable one (i.e. it is able to form a one-to-one correspondence with the positive integers). As an example, let us suppose that one of the agents that belong to the agent community $\mathrm{AgP}$, at the registration phase, does not specify its interest topics; it just informs that it has interest topics. This means that the interest topics of the agent are unknown (Program 2).

$\neg$ skill $(A, B) \leftarrow$ not skill $(A, B) \wedge$ not exceptiontskill(A,B).

exceptionskill $(A, B) \leftarrow$ skill $(A$, something).

skill('John', something).

Program 2. Information related to the agent interest topics. 
Another type of null value denotes information of an enumerated set. Following the previous example, suppose that an agent does not give information related to its availability, but its state of affairs is one of the three: uncommitted, committed or in_action (Program 3).

$\neg$ availability $(A, B) \leftarrow$ not availability $(A, B) \wedge$ not exceptionavailability $(A, B)$.

exceptionavailability('John',committed).

exceptionavailability('John', uncommitted).

exceptionavailability('John', in_action).

$\neg(($ exceptionavailability ('John',A) $\vee$ exceptionavailability('John', B) $) \wedge \neg$ (exceptionavailability('John',A) ^ exceptionavailability('John',B)).

/* This invariant denotes that the agent states of committed, uncommitted and in_action are disjointed */

Program 3. Information related to the agent's availability.

\subsection{Quality of Information of the Agent`s Profile}

The quality of information about an asset $K$ (given in terms of the quality of information that emerges from the extension of predicate $K$ ) is given by $Q_{K}=1 / \mathrm{Card}$, where Card denotes the cardinality of the exception set for predicate $K$, being $K$ disjunctive. One the other hand, if $K$ contradicts disjunction,

$$
\left(\begin{array}{c}
\text { Card } \\
1
\end{array}\right)+\left(\begin{array}{c}
\text { Card } \\
2
\end{array}\right)+\ldots+\left(\begin{array}{c}
\text { Card } \\
\text { Card }
\end{array}\right) \text { will stand for the cardinality of the combined set }
$$

of terms that make the extension of predicate $K$ (with respect to a particular asset $K$ ) (where $\left(\begin{array}{c}\text { Card } \\ p\end{array}\right)=\frac{\text { Card! }}{p !(\text { Card }-p) !}$ gives the number of combinations of Card elements, taken $p$ to $p$, where $o \leq p \leq$ Card). $Q_{K}$ is, in this case, given in the form:

$$
Q_{k}=\frac{1}{\left(\begin{array}{c}
\text { Card } \\
1
\end{array}\right)+\left(\begin{array}{c}
\text { Card } \\
2
\end{array}\right)+\ldots+\left(\begin{array}{c}
\text { Card } \\
\text { Card }
\end{array}\right)}
$$

The quality of the information that the system detains about agent AgPj, may now be defined as follows [Marreiros, 2006]: 


$$
Q^{A g P_{i}}\left(\text { Profile }_{A g P_{j}}\right)=\frac{\sum_{k=1}^{N} Q_{k}^{A g P_{j}} * W_{k}^{A g P_{i}}}{\sum_{k=1}^{N} W_{k}^{A g P_{i}}}
$$

where $N$ stands for the number of assets of the profile, $Q_{k}^{A g P j}$ is the quality measure of $\mathrm{K}$ and $W_{k}^{A g P i}$ denotes the contribution of $\mathrm{K}$ to the agent's profile. It is now possible, based on a set of (evolving) Decision Trees (DTs), to follow, day in, day out, the elderly. However, DTs are not simple representations of a decision making process, they may also apply to categorization problems, i.e., for example, instead of saying that one wish to represent a DT to plan what to do, on a weekend,

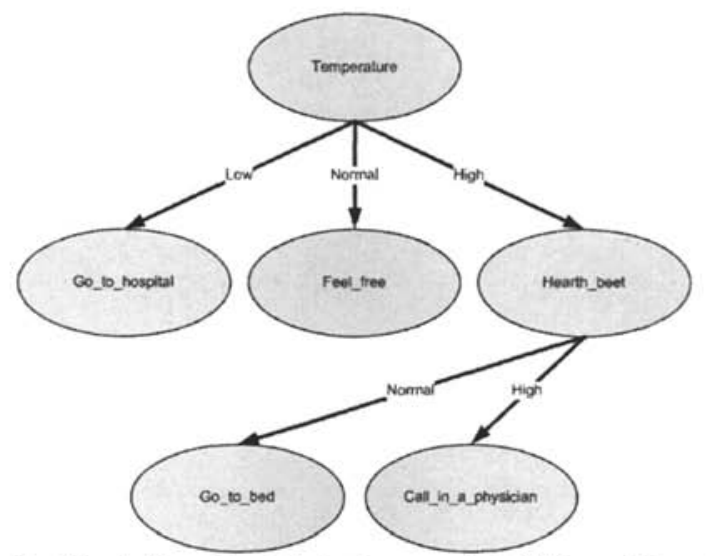

we could ask what kind of weekend is to be expected. This could easily be phrased as a question of learning a DT to decide in which category a weekend fits in (e.g., if it rains and it is windy then it is a weekend not to be remembered).

Fig 2.Decision Tree to watch the Elderly State of Health

One may now look to the process of DTs construction (e.g., to decide what to do at the weekend). One may use some background information as axioms and deduce what to do (e.g. one can know that the family is in town and that they like going to the cinema). Then, using, for example, Modus Ponens, we may decide to go to the cinema. Another way to stand around, it is by generalizing from previous experiences (e.g. let us consider all the times we had a really good weekend). If this is the case, one is using an inductive, rather than deductive method to construct the DTs (in this case one is using, for example, Modus Mistakens). Therefore, one may have Figure 2 and Figure 3.

On the other hand, there is a link between decision tree representations and logical representations, which can be exploited to make it easier to understand (to read) learned DTs. If we think better about it, every DT is actually a disjunction of implications (i.e., if ... then statements), and the implications are Horn clauses, i.e. a conjunction of terms implying a single term (Program 4). 


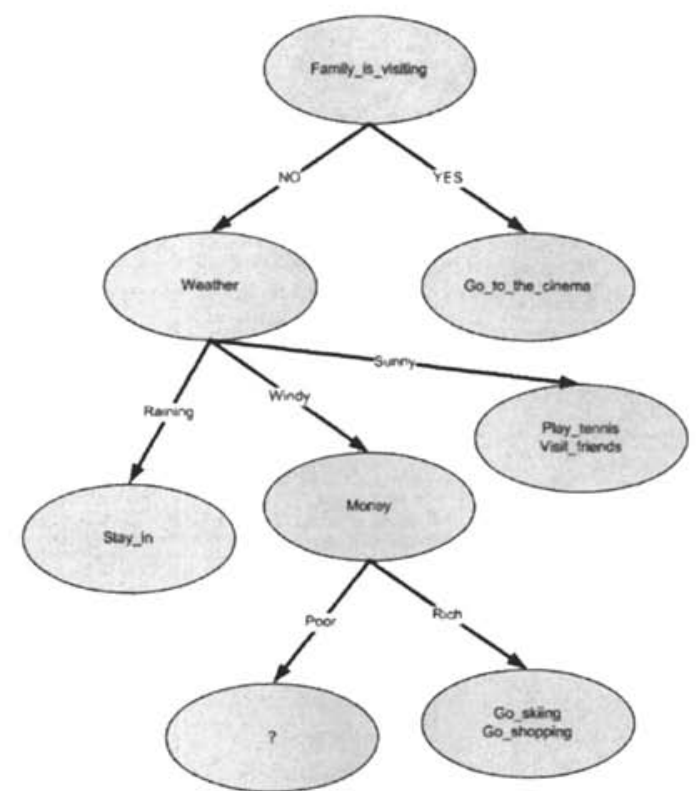

Fig 3. Decision Tree to gaze at the Elderly Weekend Planning

if family is visiting then go-to-the-cinema $\vee$ if $\neg$ family_is_visiting $\wedge$ weather_is_sunny then play_tenis $\wedge$ visit_friends $\vee$ ...

Program 4 - Reading from the root node to each leaf node.

The DTs depicted in Figures 2 and 3 may now be given in terms of logic programs or theories. For Figure 3 one may have (Program 5):

if family is_visiting then go-to-the-cinema.

if $\neg$ family_is_visiting $\wedge$ weather_is(weather, sunny) then play_tenis.

if $\neg$ family_is_visiting $\wedge$ weather_is(weather, sunny) then visit_friends.

...

if $\neg$ family_is_visiting $\wedge$ weather_is(weather, windy) $\wedge$ money_is(money, rich) then go_shopping.

if $\neg$ family_is_visiting $\wedge$ weather_is(weather, windy) $\wedge$ money_is(money, rich) then go_skiing.

$\neg($ (go_shopping $\vee$ go_skiing $) \wedge \neg$ (go_shopping $\wedge$ go_skiing) $)$.

/* This invariant denotes that the options of going to shop or going to skiing are disjointed */

$\cdots$

family_is_visiting.

$\neg$ family_is_visiting. 
$\neg($ family_is_visiting $\vee(\neg$ family_is_visiting $)) \wedge \neg$ (family_is_visiting $\Lambda(\neg$ family_is_visiting)).

/* This invariant denotes that the occurrences family_is_visiting and

$\neg$ family_is_visiting are disjointed */

$\neg$ weather_is(weather, X $) \leftarrow$

not weather_is $($ weather, $\mathrm{X}) \wedge$ not exceptionweather_is(weather, $\mathrm{X}$ ).

weather_is(weather, sunny).

weather_is(weather, windy).

weather_is(weather, raining).

$\neg$ mone_is(money, $\mathrm{X}) \leftarrow$

not money_is $($ money, $\mathrm{X}) \wedge$ not exceptionmoney_is(money, $\mathrm{X}$ ).

money_is(money, rich).

money_is(money,poor).

Program 5 - Decision Tree Meta-Logic Program to gaze at the Elderly Weekend Planning.

The call centers (i.e. the ServiceCallCenter and the CareCallCenter) may now receive from the premises under monitoring the elderly plans for the weekend, knowing also how good or bad they are, being therefore in a position to make the right judgments (Figure 4).

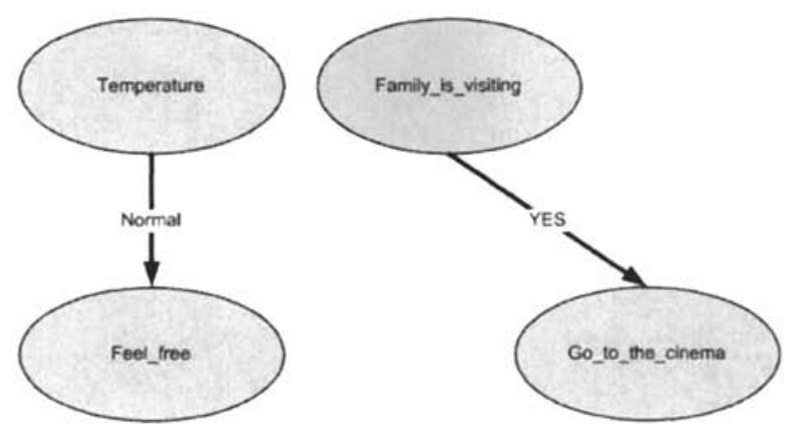

Fig 4 . Messages sent to the call centers

An advice is got in return (e.g., you may go with your family to the cinema). This advice is given not only in terms of the factual information gathered by the sensors, in terms of the DT or corresponding logical formulae depicted in Figure 2, but also attending to an evaluation of the quality of information of such formulae [8]. 


\section{Conclusion}

Based on a set of evolving DTs, it is possible not only to monitor the day in, day out of the elderly, but provide some useful advice. In future work, and attending to such scenarios, we may use collaborative networks as a support for different, but interconnected virtual organizations, that could provide to all the population in general, and the elderly, in particular way, a certain amount of remote services (e.g. healthcare, entertainment, learning), without delocalizing or messing up with their routine, in a more effective and intelligent way.

\section{References}

1. Giráldez, M., Casal, C.: The Role of Ambient Intelligent in the Social Integration of the Elderly. IOS Press (2005)

2. Camarinha-Matos, L.M., Afsarmanesh, H.: Virtual Communities and Elderly Support. Advances in Automation, Multimedia and Video Systems, and Modern Computer Science. WSES (2001) 279-284

3. Marreiros, G., Novais, P., Machado, J., Ramos, C., Neves, J.: An Agent-based Approach to Group Decision Simulation using Argumentation. International MultiConference on Computer Science and Information Tecnology, Workshop Agent-Based Computing III (ABC 2006), Wisla, Poland (2006) 225-232

4. Augusto, J.C., McCullah, P., McClelland, V., Walden, J.-A.: Enhanced Healthcare Provision Through Assisted Decision-Making in a Smart Home Environment. 2nd Workshop on Artificial Inteligence Techniques for Ambient Inteligence (2007)

5. Dosi, G.: "Sources, procedures and microeconomics effects of innovation". Economic Literature 26 (1998) 1120-1171

6. Malmberg, A.: "Industrial Geography: agglomeration and local milieu". Progress in Human Geography, Vol. 20 (1996) 392-403

7. Neves, J.: "A Logic Interpreter to Handle Time and Negation in Logic Data Bases". In: ACM (ed.): The Fifth Generation Challenge (1984) 50-54

8. Marreiros, G., Santos, R., Ramos, C., Neves, J., Novais, P., Machado, J., Bulas-Cruz, J. Ambient Intelligence in Emotion Based Ubiquitous Decision Making. Proceedings of the International Joint Conference on Artificial Intelligence (IJCAI 2007) - 2nd Workshop on Artificial Intelligence Techniques for Ambient Intelligence (AITAmI'07) (2007) 